\title{
The Incompleteness of Quantum Mechanics Demonstrated by Considerations of Relativistic Kinetic Energy
}

\author{
Koshun Suto \\ Chudai-Ji Temple, Isesaki, Japan \\ Email: Koshun_suto129@mbr.nifty.com
}

How to cite this paper: Suto, K. (2020) The Incompleteness of Quantum Mechanics Demonstrated by Considerations of Relativistic Kinetic Energy. Journal of Applied Mathematics and Physics, 8, 210-217. https://doi.org/10.4236/jamp.2020.82016

Received: December 18, 2019

Accepted: January 17, 2020

Published: January 20, 2020

Copyright $\odot 2020$ by author(s) and Scientific Research Publishing Inc. This work is licensed under the Creative Commons Attribution International License (CC BY 4.0).

http://creativecommons.org/licenses/by/4.0/ (c) (i) Open Access

\begin{abstract}
The energy levels of a hydrogen atom, derived by Bohr, are known to be approximations. This is because the classical quantum theory of Bohr does not take the theory of relativity into account. In this paper, the kinetic energy and momentum of an electron in a hydrogen atom are treated relativistically. A clearer argument is developed while also referring to papers published in the past. The energy levels of a hydrogen atom predicted by this paper almost match the theoretical values of Bohr. It is difficult to experimentally distinguish the two. However, this paper predicts the existence of an $n=0$ energy level that cannot be predicted even with Dirac's relativistic quantum mechanics. The only quantum number treated in this paper is $n$. This point falls far short of a finished quantum mechanics. However, even in discussion at the level of this paper, it can be concluded that quantum mechanics is an incomplete theory.
\end{abstract}

\section{Keywords}

Einstein's Energy-Momentum Relationship, Relativistic Kinetic Energy, $n=0$ Energy Level, Dirac's Relativistic Wave Equation

\section{Introduction}

Quantum mechanics is a physical theory representative of the 20th century, together with Einstein's theory of relativity. Einstein devised various thought experiments, such as the EPR paradox, and thereby attempted to demonstrate the incompleteness of quantum mechanics. Bohr, however, defended quantum mechanics by fending off all of Einstein's challenges. At present, there is no one objecting to quantum mechanics among physicists regarded as orthodox. The behavior of matter in the natural world is different than Einstein imagined. Eins- 
tein believed that things like non-local phenomena do not exist in the natural world.

However, phenomena which violate Bell's theorem exist in the nature. In the beginning, violations of Bell's theorem were thought to signify the instantaneous transmission of information. However, today it is known that violations of Bell's theorem are due to quantum entanglement.

Bohr fended off Einstein's challenges, but that does not mean the completeness of quantum mechanics has been shown. Even if a theory can be regarded as perfect and faultless, its authority will be lost with even a single discrepancy.

Einstein's special theory of relativity is a theory relating to physical quantities possessed by nature (reality) which exists with no relation to human beings. In the micro world, in contrast, the state of an object is changed by observation. Observed physical quantities are the values of states after a change due to observation. Quantum mechanics is not a theory which predicts the values of physical quantities possessed by reality prior to observation.

Quantum mechanics is a theory for finding the mathematical regularities between observed physical quantities. Einstein had doubts about this theoretical structure of quantum mechanics, typified by Heisenberg's matrix mechanics.

This paper discusses the relativistic kinetic energy of an electron in a hydrogen atom.

Einstein's energy-momentum relationship holds for isolated systems in free space. There is another relationship applicable to an electron in a hydrogen atom, where potential energy is present. Using the newly derived relationship, it becomes possible to discuss relativistic kinetic energy of an electron in a hydrogen atom. In this paper, an elementary discussion is provided using only the principal quantum number $n$.

Incidentally, in classical mechanics, the kinetic energy $K$ of a mass point is given by the following formula.

$$
K=\frac{1}{2} m v^{2} .
$$

Here, $m$ is the mass of the mass point. In classical mechanics, the mass of a mass point does not depend on the velocity of the mass point, and thus there is no distinction between rest mass $m_{0}$ and relativistic mass $m$. However, the two must be distinguished when handling mass relativistically. To prevent confusion in this paper, $m$ in Equation (1) is set to $m_{0}$ beforehand.

Also, if the classical kinetic energy in Equation (1) is expressed as $K_{\mathrm{cl}}$, then Equation (1) can be written as follows (The "cl" in $K_{\mathrm{cl}}$ is an abbreviation for "classical”).

$$
K_{\mathrm{cl}}=\frac{1}{2} m_{0} v^{2} .
$$

In addition, $K_{\mathrm{cl}}$ can also be written also follows using classical momentum $p_{\mathrm{cl}}$.

$$
K_{\mathrm{cl}}=\frac{p_{\mathrm{cl}}^{2}}{2 m_{0}}, \quad p_{\mathrm{cl}}=m_{0} v .
$$


The next section discusses relativistic kinetic energy $K_{\mathrm{re}}$ and momentum $p_{\mathrm{re}}$. (The "re" in $K_{\mathrm{re}}$ and $p_{\mathrm{re}}$ stands for "relativistic").

\section{Relativistic Kinetic Energy and Momentum}

Among the formulas typical of STR, there is the following energy-momentum relationship.

$$
\left(m_{0} c^{2}\right)^{2}+p^{2} c^{2}=\left(m c^{2}\right)^{2} .
$$

$m_{0} c^{2}$ is rest mass energy, and $m c^{2}$ is relativistic energy. Also, $p$ is relativistic momentum (The significance of calling $p$ "relativistic" is described later).

Now, Equation (4) can be rewritten as follows.

$$
\begin{aligned}
& p_{\mathrm{re}}^{2}=(m c)^{2}-\left(m_{0} c\right)^{2} \\
= & \left(m+m_{0}\right)\left(m c^{2}-m_{0} c^{2}\right) .
\end{aligned}
$$

A. Sommerfeld once defined kinetic energy as the difference between the relativistic energy $m c^{2}$ and rest mass energy $m_{0} c^{2}$ of an object [1]. That is,

$$
K_{\mathrm{re}}=m c^{2}-m_{0} c^{2} .
$$

If this definition is used, then Equation (5b) becomes as follows.

$$
p_{\mathrm{re}}^{2}=\left(m_{0}+m\right) K_{\mathrm{re}} .
$$

Therefore,

$$
K_{\mathrm{re}}=\frac{p_{\mathrm{re}}^{2}}{m_{0}+m} .
$$

Equation (8) is the formula for relativistic kinetic energy.

Next, let's consider the significance of $p_{\mathrm{re}}$ in Equation (8). According to STR,

$$
m=\frac{m_{0}}{\left(1-v^{2} / c^{2}\right)^{1 / 2}} .
$$

If Equation (9) is substituted into Equation (5a), the following equation is obtained.

$$
p_{\mathrm{re}}^{2}=\left[\frac{m_{0} c}{\left(1-v^{2} / c^{2}\right)^{1 / 2}}\right]^{2}-\left(m_{0} c\right)^{2} .
$$

Rearranging, we obtain

$$
\begin{aligned}
p_{\mathrm{re}}^{2} & =\left(m_{0} c\right)^{2}\left(\frac{1}{1-v^{2} / c^{2}}-1\right) \\
& =\left(m_{0} v\right)^{2}\left(\frac{c^{2}}{c^{2}-v^{2}}\right) \\
& =\frac{m_{0}^{2}}{\left(1-v^{2} / c^{2}\right)} v^{2}
\end{aligned}
$$




$$
=m^{2} v^{2}
$$

From this,

$$
p_{\text {re }}=m v \text {. }
$$

In the two formulas for momentum (3) and (12), caution is necessary because the masses in the formulas are different.

Incidentally, there is no concept of relativistic mass in classical mechanics. If we assume $m=m_{0}$, then the formula for relativistic kinetic energy matches the formula for kinetic energy in classical mechanics. That is,

$$
K_{\mathrm{re}}=\frac{p_{\mathrm{re}}^{2}}{m_{0}+m}=\frac{m^{2} v^{2}}{m_{0}+m} \quad \rightarrow \quad \frac{m_{0}^{2} v^{2}}{2 m_{0}}=\frac{p_{\mathrm{cl}}^{2}}{2 m_{0}}=K_{\mathrm{cl}} .
$$

\section{Comparison of the Energy Levels of Bohr and This Paper}

According to the virial theorem, the following relation holds between $K$ and $V$ :

$$
\langle K\rangle=-\frac{1}{2}\langle V\rangle \text {. }
$$

The average time of $K$ is equal to $-1 / 2$ the time average of $V$. Also, the sum of the time average $K$ and the time average of the total mechanical energy $E$ of the entire system becomes 0 . That is,

$$
\langle K\rangle+\langle E\rangle=0 \text {. }
$$

Next, if Equation (14) and Equation (15) are combined, the result is as follows:

$$
\langle E\rangle=-\langle K\rangle=\frac{1}{2}\langle V\rangle .
$$

Bohr thought the following quantum condition was necessary to find the energy levels of the hydrogen atom.

$$
m_{\mathrm{e}} v_{n} \cdot 2 \pi r_{n}=2 \pi n \hbar
$$

Here,

$$
\hbar=\frac{h}{2 \pi}=\frac{m_{\mathrm{e}} c \lambda_{\mathrm{C}}}{2 \pi} .
$$

Next, when $r_{\mathrm{e}} / \alpha$ is found,

$$
\frac{r_{\mathrm{e}}}{\alpha}=\frac{e^{2}}{4 \pi \varepsilon_{0} m_{\mathrm{e}} c^{2}} \frac{4 \pi \varepsilon_{0} \hbar c}{e^{2}}=\frac{\lambda_{\mathrm{C}}}{2 \pi} .
$$

Here, $r_{\mathrm{e}}$ is the classical electron radius and $\alpha$ is the fine structure constant. Each of these can be written as follows.

$$
\begin{aligned}
& r_{\mathrm{e}}=\frac{e^{2}}{4 \pi \varepsilon_{0} m_{\mathrm{e}} c^{2}} . \\
& \alpha=\frac{e^{2}}{4 \pi \varepsilon_{0} \hbar c} .
\end{aligned}
$$

Also, the radius of the electron orbital derived by Bohr can be rewritten as follows. 


$$
r_{n}=4 \pi \varepsilon_{0} \frac{\hbar^{2}}{m e^{2}} n^{2}=\frac{e^{2}}{4 \pi \varepsilon_{0} m_{\mathrm{e}} c^{2}}\left(\frac{4 \pi \varepsilon_{0} \hbar c}{e^{2}}\right)^{2} n^{2}=\frac{r_{\mathrm{e}}}{\alpha^{2}} n^{2}=\frac{\lambda_{\mathrm{C}}}{2 \pi \alpha} n^{2} .
$$

If the value of $r_{n}$ in Equation (22) is substituted into Equation (16),

$$
m_{\mathrm{e}} v_{n} \frac{\lambda_{\mathrm{C}}}{\alpha} n^{2}=m_{\mathrm{e}} c \lambda_{\mathrm{C}} n
$$

Using this,

$$
\frac{v_{n}}{c}=\frac{\alpha}{n} .
$$

Equation (24) is the core part of Bohr's quantum condition (17) [2]. This condition is non-relativistically correct, but relativistically it is incorrect. However, Equation (24) is also valid when deriving a relativistic formula.

When both sides of Equation (24) are squared, and then multiplied by $m_{\mathrm{e}} / 2$,

$$
\frac{1}{2} \frac{m_{\mathrm{e}} v_{n}^{2}}{c^{2}}=\frac{1}{2} \frac{m_{\mathrm{e}} \alpha^{2}}{n^{2}}
$$

Hence,

$$
E_{\mathrm{BO}, n}=-K_{\mathrm{cl}, n}=-\frac{1}{2} m_{\mathrm{e}} v_{n}^{2}=-\frac{\alpha^{2} m_{\mathrm{e}} c^{2}}{2 n^{2}}=-\frac{1}{2}\left(\frac{1}{4 \pi \varepsilon_{0}}\right)^{2} \frac{m_{\mathrm{e}} e^{4}}{\hbar^{2}} \cdot \frac{1}{n^{2}}, \quad n=1,2, \cdots
$$

Bohr thought that the kinetic energy of an electron constituting an atom was $(1 / 2) m_{\mathrm{e}} v_{n}^{2}$. However, to derive more precise energy levels, the relativistic kinetic energy of the electron must be treated as a problem.

The author has previously derived the following relationships applicable to the electron constituting a hydrogen atom [3].

$$
\left(m_{n} c^{2}\right)^{2}+p_{n}^{2} c^{2}=\left(m_{\mathrm{e}} c^{2}\right)^{2}
$$

Here, $m_{n}$ is the mass of an electron in a state where the principal quantum number is $n$.

Also, the relativistic kinetic energy $K_{\mathrm{re}, n}$ of an electron in a hydrogen atom can be defined as follows.

$$
K_{\mathrm{re}, n}=m_{0} c^{2}-m_{n} c^{2} .
$$

Therefore, when Equation (16) is taken into account, the energy levels $E_{\mathrm{SU}, n}$ of a hydrogen atom are as follows.

$$
E_{\mathrm{SU}, n}=-K_{\mathrm{re}, n}=m_{n} c^{2}-m_{0} c^{2} .
$$

Here, $E_{\mathrm{SU}, n}$ is the energy levels of a hydrogen atom predicted by Suto.

Incidentally, it is known that the following relationship can be derived from Equation (4).

$$
m=m_{0}\left(1-\frac{v^{2}}{c^{2}}\right)^{-1 / 2}
$$

If the method which derived Equation (30) is also applied to Equation (27), the following relationship can be derived. 


$$
m_{n}=m_{\mathrm{e}}\left(1+\frac{v_{n}^{2}}{c^{2}}\right)^{-1 / 2} .
$$

However, the discreteness of the quantum world is not incorporated into Equation (31). Thus, Equation (24) is applied, and Equation (31) is changed to the following quantum theoretic formula.

$$
m_{n}=m_{\mathrm{e}}\left(1+\frac{\alpha^{2}}{n^{2}}\right)^{-1 / 2} .
$$

If Equation (32) is substituted into Equation (29), the following formula is obtained.

$$
\begin{gathered}
E_{\mathrm{SU}, n}=m_{\mathrm{e}} c^{2}\left[\left(1+\frac{\alpha^{2}}{n^{2}}\right)^{-1 / 2}-1\right] \\
=m_{\mathrm{e}} c^{2}\left[\left(\frac{n^{2}}{n^{2}+\alpha^{2}}\right)^{1 / 2}-1\right], n=0,1,2, \cdots
\end{gathered}
$$

what should be noted here is that Equation (33) has an $n=0$ energy level. This state is also not included in the solutions of the relativistic wave equation of $\mathrm{Di}$ rac.

The existence of an $n=0$ energy level in the hydrogen atom has already been predicted in a past paper [4]. However, in References [4], Equation (33) was derived by assuming Equation (24). In the present case, success has been achieved in logically deriving Equation (24).

Here, if the part in parentheses in Equation (33a) is developed as a Taylor series,

$$
\begin{gathered}
E_{\mathrm{SU}, n}=m_{\mathrm{e}} c^{2}\left[\left(1-\frac{\alpha^{2}}{2 n^{2}}+\frac{3 \alpha^{4}}{8 n^{4}}-\frac{5 \alpha^{6}}{16 n^{6}}+\cdots\right)-1\right] \\
\approx-\frac{\alpha^{2} m_{\mathrm{e}} c^{2}}{2 n^{2}} .
\end{gathered}
$$

From this, it is evident that Equation (26) derived by Bohr is an approximation of Equation (33).

Next, the following table summarizes the energies of a hydrogen atom obtained from Equation (26) and Equation (33) (Table 1).

The following values of CODATA were used when calculating energies.

$$
\begin{gathered}
\alpha=7.2973525693 \times 10^{-3} . \\
c=2.99792458 \times 10^{8} \mathrm{~m} \cdot \mathrm{s}^{-1} . \\
m_{\mathrm{e}}=9.1093837015 \times 10^{-31} \mathrm{~kg} .
\end{gathered}
$$

\section{Conclusions}

The following table summarizes formulas of physical quantities derived from classical theory and relativistic arguments (Table 2). 
Table 1. Comparison of the energies of a hydrogen atom predicted by Bohr's classical quantum theory and this paper.

\begin{tabular}{ccc}
\hline & Bohr's Energy Levels & This Paper \\
\hline$n=0$ & - & $-0.511 \mathrm{MeV}\left(-m_{\mathrm{c}} c^{2}\right)$ \\
1 & $-13.60569 \mathrm{eV}$ & $-13.60515 \mathrm{eV}$ \\
2 & $-3.40142 \mathrm{eV}$ & $-3.40139 \mathrm{eV}$ \\
3 & $-1.511744 \mathrm{eV}$ & $-1.511737 \mathrm{eV}$
\end{tabular}

Table 2. Classical formulas (Classical mechanics and classical quantum theory) and formulas derived in this paper for physical quantities.

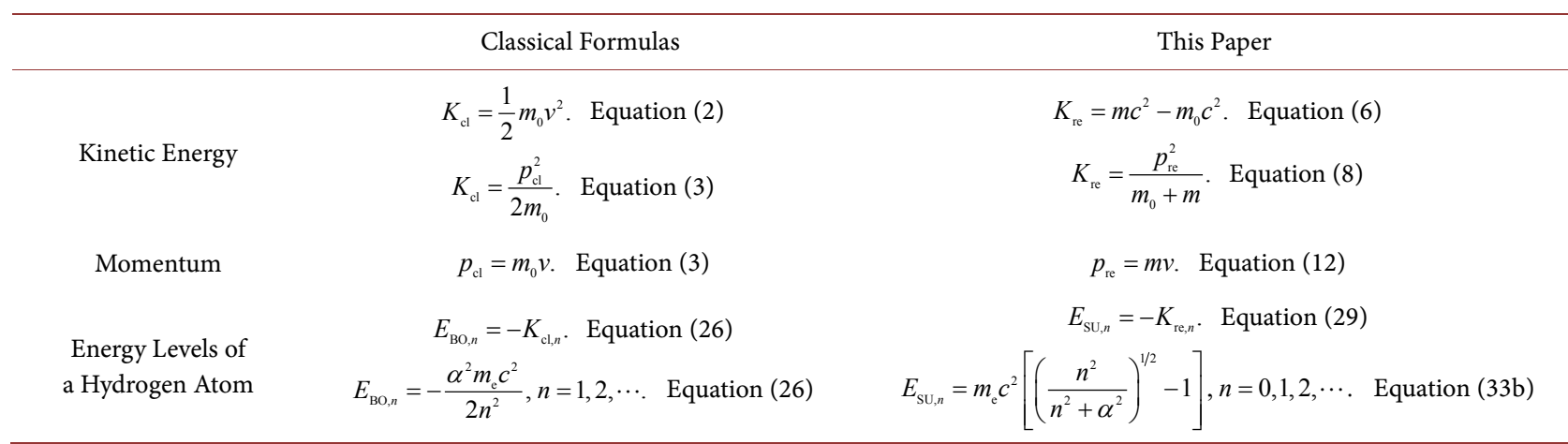

The energies $E_{\mathrm{SU}, n}$ and $E_{\mathrm{BO}, n}$ approach each other, and their values are difficult to experimentally distinguish. However, the decisive difference between the two is the existence of an $n=0$ energy level. This paper has discussed cases including only the quantum number $n$.

This point falls far short of a finished quantum mechanics. Finding more exact energy levels will require to solve the relativistic wave equation derived by the author [5] [6].

However, even in discussion at the level of this paper, it can be concluded that quantum mechanics is an incomplete theory.

\section{Acknowledgements}

I would like to express my thanks to the staff at ACN Translation Services for their translation assistance.

\section{Conflicts of Interest}

The author declares no conflicts of interest regarding the publication of this paper.

\section{References}

[1] Sommerfeld, A. (1923) Atomic Structure and Spectral Lines, Methuen \& Co. Ltd., London, 528.

[2] Suto, K. (2019) The Relationship Enfolded in Bohr's Quantum Condition and a Previously Unknown Formula for Kinetic Energy. Applied Physics Research, 11, 
19-34. https://doi.org/10.5539/apr.v11n1p19

[3] Suto, K. (2011) An Energy-Momentum Relationship for a Bound Electron Inside a Hydrogen Atom. Physics Essays, 24, 301-307. https://doi.org/10.4006/1.3583810

[4] Suto, K. (2014) $n=0$ Energy Level Present in the Hydrogen Atom. Applied Physics Research, 6, 109-115. https://doi.org/10.5539/apr.v6n5p109

[5] Suto, K. (2014) Previously Unknown Ultra-Low Energy Level of the Hydrogen Atom Whose Existence Can Be Predicted. Applied Physics Research, 6, 64-73. https://doi.org/10.5539/apr.v6n6p64

[6] Suto, K. (2018) Derivation of a Relativistic Wave Equation More Profound Than Dirac's Relativistic Wave Equation. Applied Physics Research, 10, 102-108. https://doi.org/10.5539/apr.v10n6p102 